\title{
Isotermas de dessorção de pimentão verde e energia envolvida no processo
}

Desorption isotherms of green bell peppers and the energy involved in the process

\section{Autores | Authors}

Tales Prado ALVES

Hequel Donizete FÓZ

Universidade Tecnológica Federal do Paraná (UTFPR)

Departamento de Processos Químicos Apucarana/PR - Brasil e-mail: talles_pa@hotmail.com hequelfoz@gmail.com

* Joel Fernando NICOLETI

Universidade Tecnológica Federal do Paraná (UTFPR)

Departamento de Processos Químicos Rua Marcílio Dias, 635 CEP: 86812-460

Apucarana/PR - Brasil e-mail:nicoleti@utfpr.edu.br

*Autor Correspondente / Corresponding Author

Recebido: Out. 01, 2014

Aprovado: Maio 18, 2015

\section{Resumo}

Este trabalho teve como objetivos a obtenção das isotermas de dessorção do pimentão verde e a avaliação das propriedades termodinâmicas de sorção do fruto. As isotermas foram obtidas em função da temperatura $\left(30^{\circ} \mathrm{C}, 40^{\circ} \mathrm{C}, 50^{\circ} \mathrm{C}\right.$ e $60^{\circ} \mathrm{C}$ ) em umidades relativas entre $4,0-90,0 \%$. Os dados experimentais foram ajustados aos modelos matemáticos GAB, BET, Halsey, Henderson e Oswin para se descrever seu comportamento. Os resultados demonstram que o modelo de GAB descreveu com maior precisão as isotermas de dessorção do pimentão verde entre as faixas de atividade de água $\left(a_{w}\right)$ selecionadas, em uma temperatura fixa. O calor isostérico de sorção $\left(\mathrm{q}_{\mathrm{st}}\right)$ pôde ser determinado a partir de dados de sorção de umidade utilizando-se a derivada da equação de Clasius-Clapeyron, obtendo-se então o calor envolvido no processo de dessorção de umidade do pimentão verde fresco. A teoria da compensação entalpia-entropia ou isocinética foi aplicada aos dados experimentais, sugerindo que o processo de dessorção é conduzido pela entalpia.

Palavras-chave: Calor isostérico; Secagem; Modelo de GAB; Compensação Entálpica-Entrópica.

\section{Summary}

This study aimed to obtain the desorption isotherms of green bell peppers and evaluate the thermodynamic properties of the moisture sorption of the fruit. The isotherms were obtained as a function of temperature $\left(30^{\circ} \mathrm{C}, 40^{\circ} \mathrm{C}, 50^{\circ} \mathrm{C}\right.$ and $60{ }^{\circ} \mathrm{C}$ ) between 4.0 and $90.0 \%$ of relative humidity. The experimental data were fitted to the GAB, BET, Halsey, Henderson and Oswin mathematical models to describe their behaviour. The results showed that the GAB model described the sorption isotherms of green bell peppers with greater accuracy in the range of water activities (aw) selected, at a fixed temperature. The isosteric sorption heat (qst) could be determined from the moisture sorption data using the derivative of the Clasius-Clapeyron equation, to obtain the heat involved in the desorption process of the moisture of fresh green bell peppers. The enthalpy-entropy compensation or isokinetic theory was applied to the experimental data and suggested that the desorption process was driven by enthalpy.

Key words: Isosteric heat; Drying; GAB model; Enthalpy-Entropy compensation. 


\section{Introdução}

No Brasil, o pimentão é uma das espécies do grupo das hortaliças mais difundidas e populares na culinária, devido ao seu sabor pungente característico, textura firme e coloração bastante intensa e brilhante, característica muito interessante na elaboração de diversos pratos e formulações. É considerada uma das dez espécies de maior importância econômica no mercado brasileiro de hortaliças (MARTINS, 2012). Sua grande produção é favorecida pelo clima e solo brasileiros que, em combinação com as tecnologias agrícolas empregadas no cultivo, garantem produtos de alta qualidade e uma grande produtividade. A produção brasileira de pimentão em 2006 foi de aproximadamente 251 mil toneladas, concentrando-se nas regiões sudeste, com 55\%, nordeste, com $27 \%$, sul, com $12 \%$, e norte, próximo a $1 \%$, com produtividade média de até 200 ton/ha, sendo a região sudeste a maior consumidora (IBGE, 2014). A alta taxa de produção é interessante para suprir a demanda interna do consumo do alimento fresco mas, por outro lado, pode haver um aumento na produção excedente, levando a desperdício, agravado devido à alta perecibilidade das frutas e hortaliças in natura, além das técnicas inadequadas de colheita e pós-colheita e manuseio até a chegada do produto ao consumidor final (FERREIRA et al., 2008).

No rol dos alimentos da dieta humana e na sua diversificação, os pimentões (Capsicum annuum L.) frescos geralmente são consumidos diretamente como um alimento ou como condimento na culinária doméstica. Seu estado in natura apresenta-se como uma ótima fonte de diversos nutrientes imprescindíveis e essenciais na manutenção diária do nosso organismo, como minerais, fibras e vitaminas, além de apresentar altos teores de compostos bioativos como quercetina, luteolina, ácido fenólico e ascórbico (HOWARD et al., 2000; MARIN et al., 2004; MATSUFUJl et al., 2007), compostos esses que possuem efeitos preventivos de doenças futuras (LIU et al., 2008). Entretanto, devido ao seu alto conteúdo de umidade, esse alimento é muito perecível, por apresentar uma atividade de água $\left(\mathrm{a}_{\mathrm{w}}\right)$ elevada, sendo de fundamental importância o estudo e pesquisa sobre sua estabilidade e conservação.

De acordo com Telis et al. (2000), os dados de isotermas de sorção e calor isostérico de sorção são importantes para vários processos, entre eles secagem, estocagem e empacotamento. Eles fornecem informação sobre o mecanismo de sorção e a interação entre os componentes do alimento e a água, além de estabelecer o conteúdo final de umidade e permitir a estimativa de energia requerida no processo de secagem.

Equações empíricas e semiempíricas têm sido propostas para correlacionar o conteúdo de umidade de equilíbrio com a atividade de água de produtos alimentícios. A equação de Guggenheim-Anderson-Boer (GAB) é proposta como o modelo mais versátil na literatura e é aplicada com sucesso para vários alimentos desidratados (GABAS et al., 2007; ARÉVALO-PINEDO et al., 2006; FARAHNAKY et al., 2009; MORAES et al., 2008). A equação de GAB é baseada na teoria de Brunauer-Emmett-Teller (BET) e envolve três coeficientes, dos quais dois são expressos em função da temperatura (MAROULIS et al., 1988).

Com os dados do calor isostérico de sorção pode-se obter uma estimativa da energia requerida para os processos de secagem, uma vez que eles fornecem informação do grau de dificuldade de a molécula de água desprender-se do substrato do alimento em função do conteúdo de umidade. A evolução do calor isostérico de sorção mostra que, em umidades altas, a energia requerida para o processo de dessorção de água do interior do alimento necessita de uma energia com valor próximo ao calor latente da água de vaporização da água pura, avaliada na mesma temperatura. Por outro lado, em teores de umidade reduzidos, como acontece no final dos processos de secagem, a energia requerida passa a patamares bastante altos, com um crescimento exponencial em seus valores.

A teoria isocinética ou da compensação entálpica-entrópica originalmente aplicada por Bell (1937) está relacionada com os fenômenos físicos e químicos de sorção da água. A variação da entalpia pode fornecer uma medida da mudança de energia que ocorre com a mistura de moléculas de água com o sorvente durante o processo de sorção, enquanto a variação de entropia pode estar relacionada com a ligação ou forças de repulsão no sistema. Em relação à energia livre de Gibbs, essa pode ser um indicativo da afinidade do sorvente com a água e estabelecer um critério de espontaneidade de sorção da molécula de água (TELIS et al., 2000). Trabalhos que aplicam essa teoria podem ser encontrados em Janjai et al. (2009), Telis et al. (2000), Moraes et al. (2008), Simal et al. (2007), entre outros.

O objetivo deste trabalho foi a obtenção experimental das isotermas de dessorção de pimentão verde em função da temperatura, correlacionando esses resultados ao calor isostérico de sorção e à teoria da compensação entálpica-entrópica.

\section{Material e métodos}

Os pimentões verdes, com teores de umidade de 94,2\% (base úmida) e sólidos solúveis de $3,2^{\circ}$ Brix em média, foram adquiridos no mercado local da cidade de Apucarana, Paraná, Brasil, e conservados sob refrigeração a $5{ }^{\circ} \mathrm{C}$. Os frutos foram selecionados visualmente analisando-se cor, tamanho e grau de firmeza. 
Isotermas de dessorção de pimentão verde e energia envolvida no processo

ALVES, T. P. et al.

Para a determinação da umidade de equilíbrio do pimentão in natura foram utilizados soluções saturadas de sais $\left[\mathrm{NaOH}, \mathrm{LiCl}, \mathrm{KC}_{2} \mathrm{H}_{3} \mathrm{O}_{2}, \mathrm{MgCl}_{2}, \mathrm{~K}_{2} \mathrm{CO}_{3}, \mathrm{Mg}\left(\mathrm{NO}_{3}\right)_{2}\right.$, $\mathrm{NaNO}_{2}, \mathrm{NaCl}, \mathrm{KCl}, \mathrm{BaCl}_{2}$ ] em quatro temperaturas $30^{\circ} \mathrm{C}$, $40{ }^{\circ} \mathrm{C}, 50^{\circ} \mathrm{C}$ e $60{ }^{\circ} \mathrm{C}$ (SPIES; WOLF, 1983). As soluções salinas saturadas foram distribuídas em potes herméticos, os quais correspondiam à faixa de umidade 0,04 a 0,90 (Tabela 1). O pimentão in natura foi cortado em pedaços pequenos, de espessura entre 1 a $2 \mathrm{~mm}$, distribuído em recipientes individuais dispostos nos potes herméticos. Cada pote comportava cinco recipientes contendo a amostra. Antes do uso, os potes, recipientes e suportes foram devidamente lavados e desinfetados em água sanitária comercial. As soluções salinas foram colocadas nos potes até uma altura de cerca de $1,5 \mathrm{~cm}$ a partir do fundo, sendo adicionadas cinco gotas de formaldeído em cada pote para inibir a atividade de fungos e a deterioração das amostras com teor de umidade superior a 0,5 .
As amostras, com o peso inicial conhecido, foram colocadas sobre os suportes e os potes fechados hermeticamente, levados a estufa com temperatura controlada.

Foi realizada a pesagem periódica das amostras por um período de aproximadamente 25 a 30 dias, até atingirem peso constante e variação de peso inferior a $0,1 \%$ (0,001 $\mathrm{g} / \mathrm{g}$ sólido). O conteúdo de umidade de equilíbrio foi determinado pelo método gravimétrico em estufa de circulação forçada a $105^{\circ} \mathrm{C}$ por um período de 4 horas (CUNNIFF, 1997).

\subsection{Desenvolvimento matemático}

A Tabela 2 apresenta os modelos de GAB, BET, Oswin, Halsey e Henderson utilizados para descrever comportamento higroscópico das isotermas de dessorção de pimentões verdes nas temperaturas de $30{ }^{\circ} \mathrm{C}, 40^{\circ} \mathrm{C}$, $50{ }^{\circ} \mathrm{C}$ e $60{ }^{\circ} \mathrm{C}$.

Tabela 1. Umidades relativas de soluções salinas saturadas em função da temperatura.

\begin{tabular}{|c|c|c|c|c|}
\hline \multirow{3}{*}{ Soluções salinas } & \multicolumn{4}{|c|}{ Umidade relativa (\%) } \\
\hline & \multicolumn{4}{|c|}{ Temperaturas } \\
\hline & $30^{\circ} \mathrm{C}$ & $40^{\circ} \mathrm{C}$ & $50^{\circ} \mathrm{C}$ & $60^{\circ} \mathrm{C}$ \\
\hline $\mathrm{NaOH}$ & 6,9 & 6,6 & 5,9 & 4,5 \\
\hline $\mathrm{LiCl}$ & 11,2 & 11,1 & 11,0 & 11,0 \\
\hline $\mathrm{KC}_{2} \mathrm{H}_{3} \mathrm{O}_{2}$ & 22,3 & 20,6 & 18,9 & 17,5 \\
\hline $\mathrm{MgCl}_{2}$ & 32,4 & 31,9 & 31,4 & 30,7 \\
\hline $\mathrm{K}_{2} \mathrm{CO}_{3}$ & 43,9 & 43,2 & 43,2 & 43,2 \\
\hline $\mathrm{Mg}\left(\mathrm{NO}_{3}\right)_{2}$ & 52,6 & 50,6 & 48,9 & 47,3 \\
\hline $\mathrm{NaNO}_{2}$ & 63,5 & 61,5 & 59,9 & 59,0 \\
\hline $\mathrm{NaCl}$ & 75,6 & 75,3 & 74,6 & 74,0 \\
\hline $\mathrm{KCl}$ & 83,5 & 82,0 & 80,9 & 80,0 \\
\hline $\mathrm{BaCl}_{2}$ & 90,0 & 89,3 & 88,4 & 87,6 \\
\hline
\end{tabular}

Tabela 2. Modelos matemáticos aplicados a isotermas.

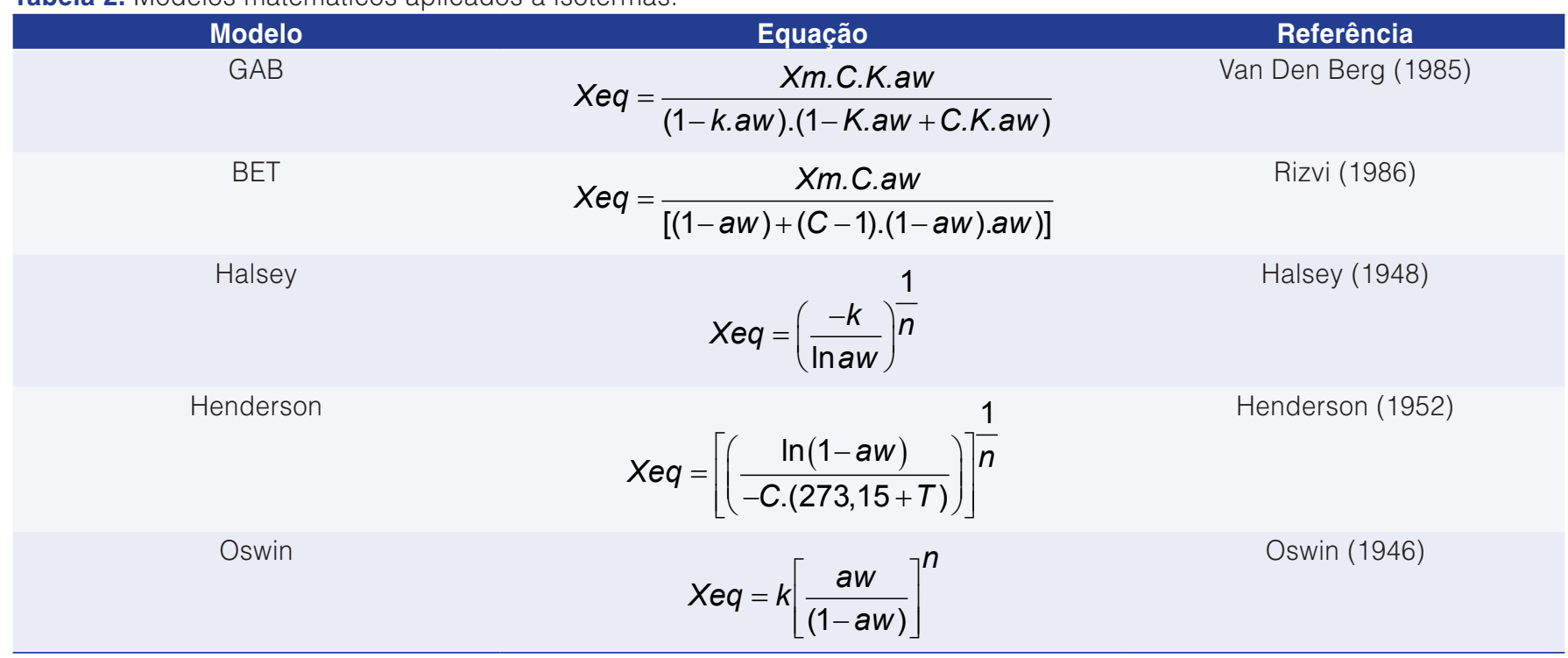


Nas equações, $X_{\text {eq }}$ corresponde ao conteúdo de umidade de equilíbrio ( $\mathrm{kg} / \mathrm{kg}$ massa seca); $X_{\mathrm{m}}$, ao conteúdo de umidade da monocamada; $a_{w}$, a atividade de água; e $\mathrm{T}$, a temperatura em ${ }^{\circ} \mathrm{C}$. As constantes dos modelos são representadas por $\mathrm{C}, \mathrm{K}, \mathrm{k}$ e $\mathrm{n}$.

A qualidade estatística dos modelos aos dados experimentais foi avaliada pelos coeficientes de determinação $\left(R^{2}\right)$, erro relativo médio (MRE) (Equação 1) e o RMS (Root Mean Square) (Equação 2).

$$
\begin{aligned}
& R M S=100 \cdot \sqrt{\frac{\sum_{i=1}^{N}(X e-X t)^{2}}{N}} \\
& M R E=\frac{100}{N} \cdot \sum_{i=1}^{N} \frac{|X e-X t|}{X e}
\end{aligned}
$$

em que $X_{t}$ e $X_{e}$ correspondem aos valores teóricos e experimentais das umidades de equilíbrio, respectivamente, e N, ao número total de pontos.

O calor isostérico de sorção, ou entalpia de sorção (qst), é definido como a diferença entre o calor isostérico de sorção total (Qst) e o calor latente de vaporização da água pura $(\lambda)$. Pode ser determinado usando-se a Equação 3, derivada da equação de Clausius-Clapeyron para um teor de umidade (X) fixo (RIZVI, 1995):

$$
\left.\frac{\partial \ln (a w)}{\partial(1 / T)}\right|_{X}=-\frac{Q s t-\lambda}{R}=-\frac{q s t}{R}
$$

em que $\mathrm{R}$ é a constante universal dos gases ideais $(8,314 \mathrm{~J} /(\mathrm{mol} . \mathrm{K}))$, T é a temperatura absoluta e $\lambda$ corresponde ao calor latente de vaporização da água pura.

A teoria da compensação entalpia-entropia é utilizada para investigar os fenômenos físicos e químicos que estão envolvidos no processo de sorção. As mudanças na entropia molar de sorção $(\mathrm{J} /(\mathrm{mol} . \mathrm{K}))$ podem ser calculadas pela equação de Gibbs-Helmholtz (4) (RIZVI, 1995):

$$
\Delta S=\frac{\Delta H-\Delta G}{T}
$$

em que $\Delta G$ é a energia livre de Gibbs ( $\mathrm{J} / \mathrm{mol})$, calculada pela Equação 5:

$$
\Delta G=R . T . \ln a w
$$

Substituindo-se a Equação 5 em 4 e rearranjando os termos, temos a Equação 6:

$$
\ln a w=\frac{\Delta H}{R T}-\frac{\Delta S}{R}
$$

A entalpia corresponde ao calor liberado ou absorvido pelo alimento a pressão constante. As mudanças ocorridas na variação de entalpia $(\Delta \mathrm{H})$ estão relacionadas com as variações energéticas devidas à interação entre água e os sólidos que constituem o alimento no processo de sorção. A variação da entropia $(\Delta S)$ é uma função de estado e está relacionada ao grau de desordem do sistema, ou seja, quanto maior for a desordem, maior a entropia do sistema. Quando o processo é espontâneo, $\Delta S$ é positivo, $\Delta H$, negativo e $\Delta G<0$. Em situação contrária, em que $\Delta \mathrm{H}$ é positivo, $\Delta \mathrm{S}$ é negativo e $\Delta \mathrm{G}>0$, tem-se que o processo não é espontâneo. Quanto maior e mais forte a interação molecular ou a força de ligação entre os compostos relacionados com a entalpia, maior será a redução na liberdade configuracional, o que leva a uma maior ordem do sistema e, consequentemente, a entropia do sistema será menor. Esse é o efeito de compensação da entalpia-entropia.

Com os valores de $\Delta \mathrm{H}$ e $\Delta \mathrm{S}$ obtidos pelo ajuste linear de $\left(\ln \mathrm{a}_{\mathrm{w}}\right)$ versus $(1 / \mathrm{T})$ repetidos para valores de umidade diferentes, esses podem ser correlacionados de acordo com a Equação 7:

$$
\Delta H=T_{B}(\Delta S)+\Delta G_{B}
$$

em que $T_{B}$ é a temperatura isocinética em (K) e $\Delta G$ é a energia livre de Gibbs a $T_{B}(\mathrm{~J} / \mathrm{mol})$.

A teoria da compensação isocinética só e válida para o processo de sorção desde que haja uma relação linear entre entalpia e entropia (MADAMBA et al., 1996; TELIS et al., 2004).

Krug et al. (1976) recomendaram um teste simples que compara a temperatura isocinética $\left(T_{B}\right)$ com a temperatura média harmônica $\left(T_{\text {hm }}\right)$ obtida pela Equação 8.

$$
T_{h m}=\frac{n}{\sum_{i=1}^{n}\left(\frac{1}{T}\right)}
$$

em que $\mathrm{n}$ é o número de temperaturas utilizadas no processo.

De acordo com Leffler (1955), se $T_{B}>T_{h m}, 0$ processo é controlado pela entalpia, enquanto que, se $T_{B}<T_{h m}$, o processo é controlado pela entropia.

\section{Resultados e discussão}

A Figura 1 apresenta as isotermas de dessorção de pimentões verdes nas temperaturas de $30{ }^{\circ} \mathrm{C}$, $40{ }^{\circ} \mathrm{C}, 50^{\circ} \mathrm{C}$ e $60^{\circ} \mathrm{C}$. Observa-se que o efeito da temperatura é mais pronunciado em atividades de água superiores a 0,60, especialmente entre as faixas de 0,80 e 0,90 , nas quais o afastamento entre as curvas é mais acentuado. Em atividades de água inferiores a 
0,6 , as curvas praticamente se sobrepõem, indicando que a temperatura não difere no comportamento das isotermas. Em atividades de água acima de 0,60, a água encontra-se fracamente ligada aos constituintes do alimento, necessitando dessa forma de energia próxima à requerida para a vaporização da água pura. Por outro

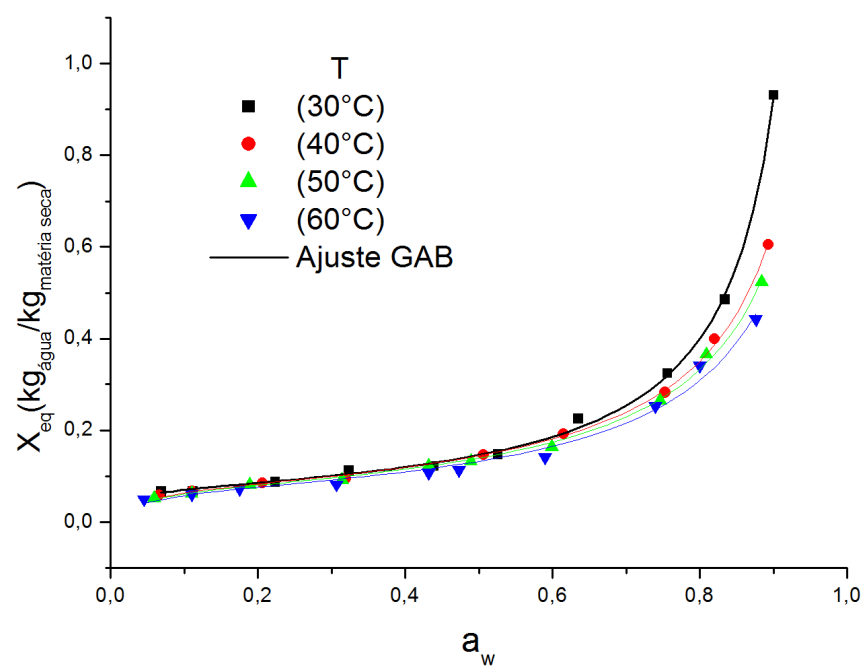

Figura 1. Isotermas de dessorção do pimentão verde. lado, conforme a atividade de água é reduzida, a água presente no alimento torna-se cada vez mais ligada ao substrato do alimento, com forças moderadas, chegando a apresentar forças de alta intensidade em atividades de água muito baixas. Desse modo, a força motriz, no caso a temperatura, necessária para a remoção da umidade em atividades de água inferior a 0,60 torna-se pouco efetiva, já que as forças de ligação das moléculas de água aos constituintes se sobressaem, necessitando assim de um incremento de temperatura muito elevado para se observar o seu efeito.

Para descrever o comportamento higroscópico do pimentão verde nas temperaturas avaliadas utilizaram-se cinco modelos - GAB, BET, Oswin, Halsey e Henderson. De acordo com a Tabela 3, o modelo de GAB apresentou os melhores ajustes, comparando-se os parâmetros estatísticos de qualidade.

Lomauro et al. (1985) relataram que as isotermas de hortaliças foram melhor descritas pelo modelo de GAB e Heldman e Hartel (2000) ressaltam que esse modelo pode ser considerado o que melhor se ajusta às isotermas dos produtos alimentícios, sendo um dos mais utilizados para tal fim.

Tabela 3. Parâmetros dos modelos ajustados às isotermas de dessorção do pimentão verde e respectivos valores de R², RMS e MRE.

\begin{tabular}{|c|c|c|c|c|c|c|c|}
\hline Modelo & $\mathrm{T}\left({ }^{\circ} \mathbf{C}\right)$ & & râmetrc & & $\mathbf{R}^{2}$ & RMS (\%) & MRE (\%) \\
\hline \multirow[t]{5}{*}{ GAB } & & C & K & $X_{m}$ & & & \\
\hline & 30 & 57,715 & 1,024 & 0,072 & 0,999 & 5,16 & 4,48 \\
\hline & 40 & 27,143 & 0,98 & 0,077 & 0,998 & 4,78 & 3,81 \\
\hline & 50 & 25,924 & 0,973 & 0,075 & 0,997 & 4,58 & 3,83 \\
\hline & 60 & 25,312 & 0,964 & 0,071 & 0,987 & 10,05 & 8,12 \\
\hline \multirow[t]{5}{*}{ BET } & & C & & $X_{m}$ & & & \\
\hline & 30 & 9,052 & & 0,089 & 0,99 & 16,97 & 12,7 \\
\hline & 40 & 85,585 & & 0,068 & 0,993 & 5,5 & 5,04 \\
\hline & 50 & 87,669 & & 0,064 & 0,99 & 6,81 & 6,02 \\
\hline & 60 & 106,545 & & 0,06 & 0,976 & 6,41 & 5,3 \\
\hline \multirow[t]{5}{*}{ Henderson } & & $\mathbf{N}$ & & C & & & \\
\hline & 30 & 0,576 & & 0,008 & 0,951 & 55,02 & 42,86 \\
\hline & 40 & 0,844 & & 0,011 & 0,96 & 40,6 & 27,38 \\
\hline & 50 & 0,914 & & 0,013 & 0,958 & 35,51 & 26,96 \\
\hline & 60 & 0,953 & & 0,014 & 0,958 & 38,7 & 26,63 \\
\hline \multirow[t]{5}{*}{ Oswin } & & $\mathbf{N}$ & & k & & & \\
\hline & 30 & 0,863 & & 0,134 & 0,979 & 38,22 & 26,99 \\
\hline & 40 & 0,654 & & 0,148 & 0,988 & 24,17 & 15,71 \\
\hline & 50 & 0,625 & & 0,144 & 0,987 & 22,31 & 15,07 \\
\hline & 60 & 0,608 & & 0,136 & 0,979 & 24,44 & 17,09 \\
\hline \multirow[t]{5}{*}{ Halsey } & & $\mathbf{N}$ & & k & & & \\
\hline & 30 & 1,011 & & 0,095 & 0,992 & 20,93 & 15,33 \\
\hline & 40 & 1,262 & & 0,06 & 0,998 & 7,77 & 5,76 \\
\hline & 50 & 1,304 & & 0,054 & 0,997 & 6,2 & 5,04 \\
\hline & 60 & 1,329 & & 0,047 & 0,988 & 6,16 & 8,43 \\
\hline
\end{tabular}

$\mathrm{X}_{\mathrm{m}}$ : umidade da monocamada $\left(\mathrm{kg}_{\text {aqua }} / \mathrm{kg}_{\text {materia seca }}\right)$; C, K, $\mathrm{k}, \mathrm{n}$ : parâmetros de ajuste; $\mathrm{R}^{2}$ : coeficiente de correlação; RMS: valor quadrático médio; MRE: erro relativo médio. 
Lewicki (1997) relata que a forma das isotermas pode ser baseada nos parâmetros $\mathrm{C}$ e $\mathrm{K}$, obtidos através do ajuste do modelo de GAB aos dados experimentais. Segundo o autor, as isotermas apresentam comportamento e forma sigmoidal, quando os valores encontrados pelo ajuste estão entre os intervalos: $0,24<\mathrm{K} \leq 1 \mathrm{e}$ $5,67 \leq \mathrm{C} \leq \infty$. Apesar de os valores dos parâmetros $\mathrm{C}$ e K obtidos estarem dentro da faixa limitada pelo autor, as isotermas de sorção de pimentão verde mostraram uma tendência mais exponencial do que sigmoidal.

Nos valores de $X_{m}$ encontrados pela equação de GAB, com exceção da menor temperatura avaliada $\left(30{ }^{\circ} \mathrm{C}\right)$, houve uma tendência de diminuição conforme aumento na temperatura. O mesmo comportamento foi observado para os parâmetros K e C. O teor de umidade $\left(\mathrm{X}_{\mathrm{m}}\right)$ corresponde à quantidade de água $\left(\mathrm{kg}_{\text {água }} / \mathrm{kg}_{\text {matéria seca }}\right)$ da monocamada, a qual não participa de reações, é muito difícil eliminá-la pela secagem e também não é congelável, por estar fortemente ligada ao produto. As constantes $\mathrm{C}$ e $\mathrm{K}$ podem ser correlacionadas com $\mathrm{O}$ calor de sorção da monocamada e das multicamadas pela equação de Arrhenius (Equações 9 e 10).

$$
\begin{aligned}
& C=C_{o} \cdot \exp \left(\frac{\Delta H_{C}}{R T}\right) \\
& K=K_{o} \cdot \exp \left(\frac{\Delta H_{K}}{R T}\right)
\end{aligned}
$$

em que $\mathrm{C}_{0}$ e $\mathrm{K}_{0}$ são constantes características das equações, $\mathrm{T}$, a temperatura absoluta $\mathrm{K}, \mathrm{R}$, a constante universal dos gases, $\Delta \mathrm{H}_{\mathrm{C}}=\left(\mathrm{H}_{\mathrm{m}}-\mathrm{H}_{\mathrm{n}}\right)$ e $\Delta \mathrm{H}_{\mathrm{K}}=\left(\lambda-\mathrm{H}_{\mathrm{n}}\right)$, sendo $\mathrm{H}_{\mathrm{m}}$ o calor total de sorção da monocamada, $\mathrm{H}_{\mathrm{n}} \mathrm{O}$ calor total de sorção das multicamadas e $\lambda$ o calor de condensação da água pura em função da temperatura.

De acordo com Telis et al. (2000), o calor isostérico de sorção é um parâmetro importante para quantificar a demanda energética no processo de dessorção.

A obtenção do calor isostérico foi calculada a partir da equação de Clausius-Clapeyron (Equação 1). A derivada do $\ln \left(a_{w}\right)$ em função do inverso da temperatura $(1 / T)$ é conseguida traçando-se um gráfico de (In $a_{w}$ versus $1 / \mathrm{T}$ ), conforme Figura 2, para diversas umidades de equilíbrio fixas. Pelo coeficiente angular do modelo linear ajustado tem-se a derivada da função, a qual, multiplicada pelo fator da constante universal dos gases $(R)$, fornece o valor do calor isostérico em função do conteúdo de umidade.

O calor isostérico de sorção (qst) em função do teor de umidade do pimentão verde é apresentado pela Figura 3. Observa-se que para conteúdos de umidades inferiores a $10 \%$ (b.s), o processo de dessorção requer uma elevação de energia substancial, caracterizado pelo acréscimo abrupto nos valores do calor isostérico. O mesmo efeito foi observado por Tolaba et al. (2004) e

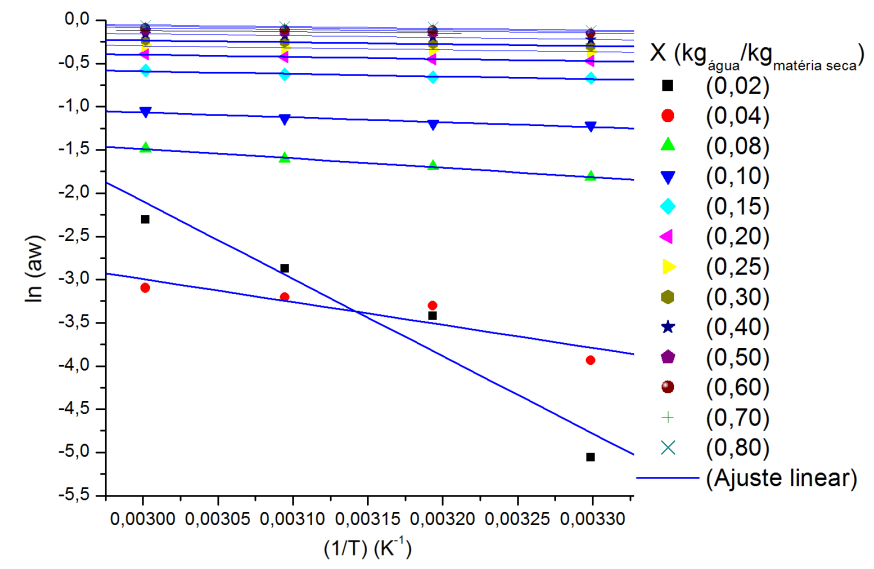

Figura 2. Relação entre $\ln \left(a_{w}\right)$ e o inverso da temperatura para obtenção do coeficiente angular e respectivo valor do calor isostérico $\left(\mathrm{q}_{\mathrm{st}}\right)$, de acordo com a Equação 3.

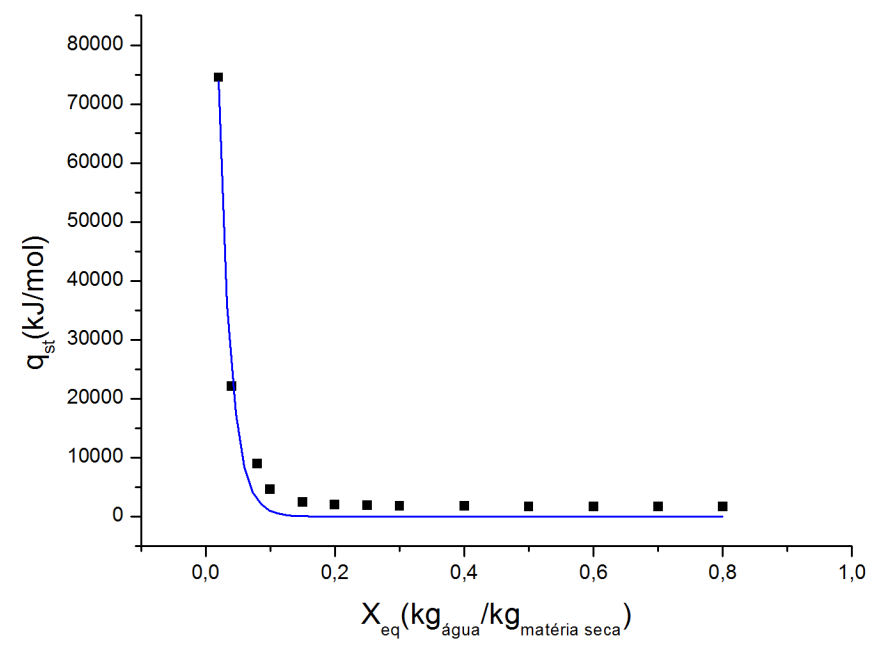

Figura 3. Calor isostérico em função do conteúdo de umidade do pimentão verde.

por Jayendra Kumar et al. (2005). Esse aumento reflete a força de interação da água com a monocamada do material. A curva sobre os pontos experimentais é o ajuste empírico exponencial (Equação 11), sendo que os valores encontrados foram $\mathrm{q}_{0}=219711,92 \mathrm{~kJ} / \mathrm{mol} \mathrm{e}$ $X_{0}=0,02 \mathrm{~kg}_{\text {agua }} / \mathrm{kg}_{\text {matéria seca }}$, com $\mathrm{R}^{2}$ superior a 0,98 .

$$
q_{s t}=q_{0} \cdot \exp \left(\frac{-X_{e q}}{X_{0}}\right)
$$

em que $\mathrm{q}_{0}$ é o calor isostérico de sorção da monocamada e $X_{0}$, o teor de umidade característico do alimento.

A teoria da compensação isocinética é válida para o processo de sorção desde que haja uma relação linear entre entalpia e entropia. Os valores de entalpia $(\Delta H)$ e de entropia $(\Delta S)$ foram determinados para diferentes conteúdos de umidade pela Equação 6. 


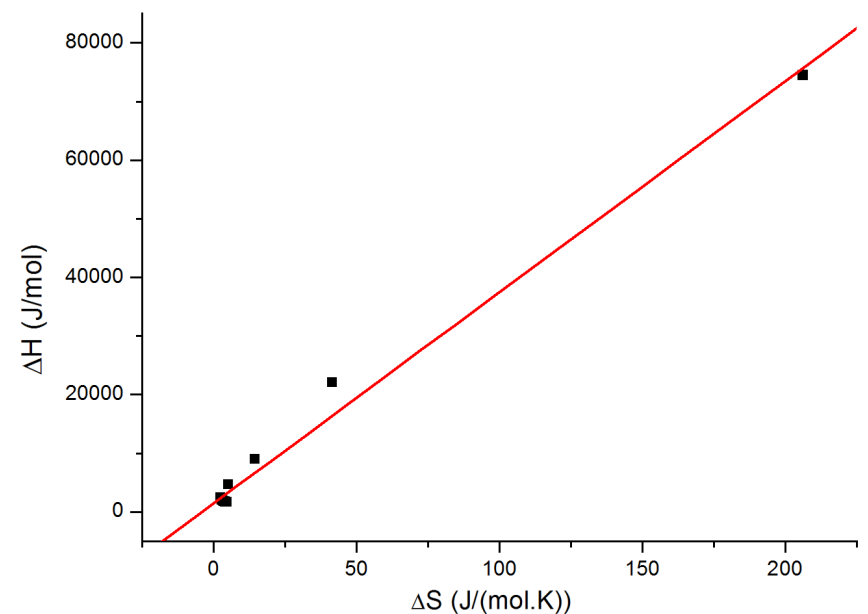

Figura 4. Variações de entalpia versus entropia no processo de dessorção do pimentão verde.

Os resultados demonstram uma relação linear entre $(\Delta \mathrm{H})$ e $(\Delta \mathrm{S})$ (Figura 4), com coeficiente de determinação $R^{2}$ superior a 0,99, satisfazendo assim as exigências da teoria da compensação isocinética.

Por meio do ajuste linear da Equação 7 foi possível obterem-se os valores da temperatura isocinética $\left(T_{B}\right)$ e da energia livre de Gibbs $\left(\Delta G_{B}\right)$ para o pimentão verde, que foram, respectivamente, $360 \mathrm{~K}$ e 1427,2 J/mol, o que caracterizou dessa forma um processo não espontâneo $\left(\Delta G_{B}>0\right)$. Telis et al. (2000) obtiveram para o processo de dessorção de caqui os seguintes valores de temperaturas isocinéticas: $467 \mathrm{~K}$ e $475 \mathrm{~K}$, respectivamente para a casca e para a polpa da fruta. Sousa et al. (2012) encontraram o valor de $T_{B}=361,22 \mathrm{~K}$ na dessorção de castanha de caju, enquanto que, para grãos de milho, Oliveira et al. (2013) reportaram o valor de 436,81 K.

A fim de avaliar a teoria da compensação entálpica-entrópica, a temperatura isocinética $\left(T_{B}\right)$ foi comparada com a temperatura harmônica $\left(T_{h m}\right)$ calculada pela Equação 8. O valor da temperatura harmônica encontrado foi de $317 \mathrm{~K}$. Uma vez que $T_{B}>$ Thm, pode-se concluir que o processo de dessorção do pimentão verde é conduzido pela entalpia (LEFFLER, 1955). De acordo com Marcinkowski (2006), quando um sistema contendo diversos compostos passa por uma transformação geralmente ocorrem alterações na entalpia e na entropia. Isso implica que quanto mais forte a interação molecular ou a força de ligação, fato esse relacionado à entalpia, maior a redução na liberdade configuracional que caracteriza uma maior ordenação do sistema, sendo essa característica própria da entropia. Dessa forma, essas duas propriedades termodinâmicas apresentam proporcionalidade, conforme sugere a teoria da compensação entalpia-entropia.

\section{Conclusões}

A equação de GAB foi a que melhor descreveu as isotermas do pimentão verde em todas as temperaturas fixadas. O calor isostérico tem seus valores aumentados conforme o conteúdo de umidade do pimentão é reduzido, um indicativo do aumento da interação das moléculas de água com os constituintes do alimento. Esse aumento no calor isostérico reflete na maior quantidade de energia necessária para se retirar água do produto, à medida que esse conteúdo de água diminui. A teoria da compensação entalpia-entropia pôde ser aplicada com sucesso e sugere que o processo de dessorção do pimentão verde é conduzido por entalpia.

\section{Agradecimentos}

Os autores agradecem à Fundação Araucária (Conv. 469/2010, Protocolo 16.742) pelo Auxílio Financeiro e pela bolsa de Iniciação Científica (PIBIQ 2012-2013).

\section{Referências}

ARÉVALO-PINEDO, A.; SANTOS, F. L.; ARÉVALO, Z. D. S.; ZUNIGA, A. D. G.; PINEDO, R. A. Desorption isotherms for murici (Byrsonima sericea) and inga (Ingá edulis) pulps. Journal of Food Engineering, Califórnia, v. 76, n. 4, p. 611-615, 2006. http://dx.doi.org/10.1016/j.jfoodeng.2005.06.011.

BELL, R. P. Relation between the energy and entropy solution and their significance. Transactions of the Faraday Society, Londres, v. 33, p. 496, 1937. http://dx.doi.org/10.1039/ tf9373300496.

CUNNIFF, P. (Ed.). Official methods of analysis of the Association of Official Analytical Chemists. 16. ed. Gaithersburg: AOAC, 1997. (v. 2).

FARAHNAKY, A.; ANSARI, S.; MAJZOOBI, M. Effect of glycerol on the moisture sorption isotherms of figs. Journal of Food Engineering, Califórnia, v. 93, n. 4, p. 468-473, 2009. http:// dx.doi.org/10.1016/j.jfoodeng.2009.02.014.

FERREIRA, M. D.; FRANCO, A. T. O.; FERRAZ, A. C. O.; CARMARGO, G. G. T.; TAVARES, M. Qualidade do tomate de mesa em diferentes etapas da fase de pós-colheita. Horticultura Brasileira, Campinas, v. 26, n. 2, p. 231-235, 2008. http://dx.doi. org/10.1590/S0102-05362008000200020.

GABAS, A. L.; TELIS, V. R. N.; SOBRAL, P. J. A.; TELIS-ROMERO, $J$. Effect of maltodextrin and arabic gum in water vapor sorption thermodynamic properties of vacuum dried pineapple pulp powder. Journal of Food Engineering, Califórnia, v. 82, n. 2, p. 246-252, 2007. http://dx.doi.org/10.1016/j.jfoodeng.2007.02.029.

HALSEY, G. Physical adsorption on noun-uniform surfaces. The Journal of Chemical Physics, New York, v. 16, n. 10, p. 931-937, 1948. 
Isotermas de dessorção de pimentão verde e energia envolvida no processo

ALVES, T. P. et al.

HELDMAN, D. R.; HARTEL, R. W. Principles of food processing. Nova Iorque: Chapman \& Hall, 2000. 288 p.

HENDERSON, S. M. A basic concept of equilibrium moisture. Agricultural Engineering, Saskatoon, v. 33, p. 29-32, 1952.

HOWARD, L. R.; TALCOTT, S. T.; BRENES, C. H.; VILLALÓN, B. Changes in phytochemical and antioxidant activity of selected pepper cultivars (Capsicum species) as influenced by maturity. Journal of Agricultural and Food Chemistry, Washington, v. 48, n. 5, p. 1713-1720, 2000. http://dx.doi.org/10.1021/jf990916t. PMid: 10820084

INSTITUTO BRASILEIRO DE GEOGRAFIA E ESTATÍSTICA IBGE. Sistema IBGE de Recuperação de Dados - SIDRA: bando de dados agregados. Rio de Janeiro: IBGE, 2014. Disponível em: <www.sidra.ibge.gov.br>. Acesso em: 22 jun. 2014.

JANJAI, S.; INTAWEE, P.; TOHSING, K.; MAHAYOTHEE, B.; BALA, B. K.; ASHARAF, M. A.; MULLER, J. Neural network modeling of sorption isotherms of longan (Dimocarpus longan Lour). Computers and Electronics in Agriculture, Nova Iorque, v. 66, n. 2, p. 209-214, 2009. http://dx.doi.org/10.1016/j. compag.2009.02.003.

JAYENDRA KUMAR, A.; SINCH, R. R. B.; PATIL, G. R.; PATEL, A. A. Effect of temperature on moisture desorption isotherms of kleer. Food Science and Technology, Campinas, v. 38, p. 303-310, 2005

KRUG, R. R.; HUNTER, W. G.; GREIGER, R. A. Enthalpy-entropy compensation. 1. Some fundamental statistical problems associated with the analysis of Van't Hoff and Arrhenius data. Journal of Physical Chemistry, Ithaca, v. 80, n. 21, p. 23352341, 1976. http://dx.doi.org/10.1021/j100562a006.

LEFFLER, J. E. The enthalpy-entropy relationship and its implications for organic chemistry. The Journal of Organic Chemistry, Washington, v. 20, n. 9, p. 1202-1231, 1955

LEWICKI, P. P. The applicability of the GAB model to food water sorption isotherms. International Journal of Food Science and Technology, Oxford, v. 32, n. 6, p. 553-557, 1997. http://dx.doi. org/10.1111/j.1365-2621.1997.tb02131.x

LIU, H.; QIU, N.; DING, H.; YAO, R. Polyphenols contents and antioxidant capacity of 68 Chinese herbals suitable for medical or food uses. Food Research International, Barking, v. 41, n. 4, p. 363-370, 2008. http://dx.doi.org/10.1016/j.foodres.2007.12.012.

LOMAURO, C. J.; BAKSHI, A. S.; LABUZA, T. P. Evaluation of food moisture isotherm equations. Part I: Fruit, vegetable and meat products. LWT - Food Science and Technology, Zurique, v. 18, n. 2, p. 111-117, 1985.

MADAMBA, P. S.; DRISCOLL, R. H.; BUCKLE, K. A. Enthalpyentropy compensation models for sorption and browning of garlic. Journal of Food Engineering, Califórnia, v. 28, n. 2, p. 109-119, 1996. http://dx.doi.org/10.1016/0260-8774(94)00072-7.
MARCINKOWSKI, E. A. Estudo da cinética de secagem, curvas de sorção e predição de propriedades termodinâmicas da proteína texturizada de soja. 2006, 127 f. Dissertação (Mestrado em Engenharia Química) - Universidade Federal do Rio Grande do Sul, Porto Alegre, 2006.

MARÍN, A.; FERRERES, F.; TOMÁS-BARBERÁN, F. A.; GIL, M. I. Characterization and quantitation of antioxidant constituents of sweet pepper (Capsicum annuum L.). Journal of Agricultural and Food Chemistry, Washington, v. 52, n. 12, p. 3861-3869, 2004. http://dx.doi.org/10.1021/jf0497915. PMid:15186108.

MAROULIS, Z. B.; TSAMI, E.; MARINOS-KOURIS, D.; SARAVACOS, G. D. Application of the GAB model to the moisture sorption isotherms for dried fruits. Journal of Food Engineering, Califórnia, v. 7, n. 1, p. 63-78, 1988. http://dx.doi. org/10.1016/0260-8774(88)90069-6.

MARTINS, W. M. O. Avaliação do pegamento e crescimento de plantas de pimentão (Capsicum annuum L.) enxertado em cultivo orgânico. Enciclopédia Biosfera, Goiânia, v. 8, n. 14, p. $149-155,2012$

MATSUFUJI, H.; ISHIKAWA, K.; NUNOMURA, O.; CHINO, M.; TAKEDA, M. Anti-oxidant content of diferente coloured sweet peppers, white, green, yellow, orange and red (Capsicum annuum L.). International Journal of Food Science \& Technology, Oxford, v. 42, n. 12, p. 1482-1488, 2007. http:// dx.doi.org/10.1111/j.1365-2621.2006.01368.x.

MORAES, M. A.; ROSA, G. S.; PINTO, L. A. A. Moisture sorption isotherms and thermodynamic properties of apple Fuji and garlic. International Journal of Food Science \& Technology, Nova Deli, v. 43, n. 10, p. 1824-1831, 2008. http://dx.doi. org/10.1111/j.1365-2621.2008.01716.x.

OLIVEIRA, D. E. C.; RESENDE, O.; SMANIOTTO, T. A. S.; SOUSA, K. A.; CAMPOS, R. C. Propriedades termodinâmicas de grãos de milho para diferentes teores de água de equilíbrio. Pesquisa Agropecuária Tropical, Goiânia, v. 43, n. 1, p. 50-56, 2013. Disponível em: <http://dx.doi.org/10.1590/S198340632013000100007>

OSWIN, C. R. The kinetics of package life III. The isotherm. Journal of the Society of Chemical Industry, London, v. 65, p. 419-421, 1946

RIZVI, S. S. H. Thermodinamic properties of foods in dehydration. In: RAO, M. A.; RIZIVI, S. S. H. (Ed.). Engineering properties of foods. New York: Marcel Dekker, 1986. p. 133-214.

RIZVI, S. S. H. Thermodynamic properties of foods in dehydration. In: RAO, M. A.; RIZVI, S. S. H. (Ed.). Engineering properties of foods. Califórnia: Academic Press, 1995. p. 223-309.

SIMAL, S.; FEMENIA, A.; CASTELL-PALOU, A.; ROSSELLO, C. Water desorption thermodynamic properties of pineapple. Journal of Food Engineering, Califórnia, v. 80, n. 4, p. 12931301, 2007. http://dx.doi.org/10.1016/j.jfoodeng.2006.10.001 
SOUSA, K. A.; RESENDE, O.; ULLMANN, R.; SMANIOTTO, T. A. S.; OLIVEIRA, D. E. C. Teoria da compensação entalpiaentropia na dessorção das sementes caju-de-árvore-do-cerrado. In: CONGRESSO DE PESQUISAS E PÓS-GRADUAÇÃO DO CÂMPUS RIO VERDE DO IF GOIANO, 1., 2012, Rio Verde. Anais... Rio Verde: Instituto Federal Goiano, 2012. p. 1-3.

SPIES, W. E. L.; WOLF, W. R. The results of the COST 90 project on water activity. In: ESCHER, F.; HALLSTROM, B.; MEFFERT, H. S.; SPIESS, W. E. L.; VOSS, G. (Ed.). Physical properties of foods. Califórnia: Applied Science Publishers, 1983. p. 65-87.

TELIS, V. R. N.; GABAS, A. L.; MENEGALLI, F. C.; TELISROMERO, J. L. Water sorption thermodynamic properties applied to persimmon skin and pulp. Thermochimica Acta, Amsterdã, v. 343, n. 1-2, p. 49-56, 2000. http://dx.doi.org/10.1016/S00406031(99)00379-2.
TELIS, V. R. N.; MURARI, R. C. B. B. D. L.; YAMASHITA, F. Diffusion coefficients during osmotic dehydration of tomatoes in ternary solutions. Journal of Food Engineering, Califórnia, v. 61, n. 2, p. 253-259, 2004. http://dx.doi.org/10.1016/S02608774(03)00097-9.

TOLABA, M. P.; PELTZER, M.; ENRIQUEZ, N.; POLLIO, M. L. Grain sorption equilibria of quinoa grains. Journal of Food Engineering, Califórnia, v. 61, n. 3, p. 365-371, 2004. http:// dx.doi.org/10.1016/S0260-8774(03)00143-2.

VAN DEN BERG, C. Development of B.E.T. like models for sorption of water of foods: theory and relevance. In: SIMATOS, D.; MULTON, J. L. (Ed). Properties of water in foods. Dordrecht: Martinus Nijhoft Publishers, 1985. p. 119-135. 